seizures (24\%), neonatal (6\%), generalized tonic-clonic (49\%), and rare complex partial. Transmission of FC showed a maternal preponderance. The phenotypic expression of the genetic basis for focal sharp waves was remarkably variable. The clinical and EEG findings suggested a multifactorial pathogenesis for epilepsies characterized by benign focal sharp waves. (Doose $\mathrm{H}$, Brigger-Heuer B, Neubauer B. Children with focal sharp waves: Clinical and genetic aspects. Epilepsia July 1997;38:788-796). (Reprints: Dr H Doose, EpilepsieZentrum, 24223 Raisdorf, Germany).

COMMENT. Focal sharp waves may be characteristic of a variety of different seizure patterns, including febrile convulsions, and not only that of rolandic epilepsy. In addition to epileptic syndromes, focal sharp waves may be found in children with learning disabilities such as dyslexia and dysphasia. The genetics of these syndromes appears to be multifactorial.

Genetics of febrile convulsions. Doose $\mathrm{H}$ and Maurer A have also reported on genetic factors in febrile convulsions. (Seizure risk in offspring of individuals with a history of febrile convulsions. Eur I Pediatr June $1997 ; 156: 476-481$ ). The risk was $10 \%$; only FC in $64 \%$ of affected offspring. Offspring of females with affected parents were at increased risk.

\title{
VAGUS NERVE STIMULATION FOR EPILEPSY
}

The Therapeutics and Technology Assessment Subcommittee of the American Academy of Neurology reports on vagus nerve stimulation (VNS) for epilepsy. A total of 104 articles and responses in 130 patients were reviewed. Data on VNS in children was sparse; 5 of 12 children with intractable partial, generalized, and mixed seizures, followed for 2 to 14 months in an open-label study, had a $90 \%$ reduction in seizure frequency. In a randomized prospective trial in 114 adult patients, high levels of stimulation showed a significant response compared to low stimulation. The degree of improvement was modest, with a mean partial seizure reduction of $31 \%$, and $39 \%$ of patients having a $>50 \%$ reduction in seizure frequency. Young patients with auras and idiopathic epilepsy had more than a $50 \%$ responder rate, while secondarily generalized seizures were not controlled. The mechanism of VNS was undetermined. Leftsided implantations were safer, since right VNS caused bradycardia. Electrodes around the vagus were connected to an infraclavicular generator pack. Serious complications were rare, but hoarseness, throat pain, and cough were common complaints during stimulation. The device is costly, and improvement in quality of life for the patient needs to be determined. (Fisher RS, Krauss GL, Ramsay E, Laxer K, Gates J. Assessment of vagus nerve stimulation for epilepsy: Report of the Therapeutics and Technology Assessment Subcommittee of the American Academy of Neurology. Neurology June 1997;49:293-297). (Reprints: Dr Robert S Fisher, Barrow Neurological Institute, 350 West Thomas Road, Phoenix, AZ 85013).

COMMENT. The panel considers vagal nerve stimulation a promising treatment for intractable partial seizures, but further controlled studies are recommended. Some predictors of a favorable response include age less than 34 years, idiopathic epilepsies, and early signs of effectiveness.

\section{KETOGENIC DIET REVISITED IN THE LABORATORY}

The effect of a ketogenic diet (KD) on kindled seizures and behavior in adult rats was studied at the Children's Hospital, Harvard Medical School, Boston, MA. In a kindling model, KD provided transient protection against 